\title{
The Effects of Scaling on High Subsonic Cavity Flow
}

\section{Oscillations and Control}

\author{
V Thangamani ${ }^{1}$, K Knowles ${ }^{2}$ and A J Saddington ${ }^{3}$ \\ Cranfield University, Defence Academy of the UK, Shrivenham, Wiltshire, SN6 8LA
}

\begin{abstract}
The effects of scaling on cavity oscillations and control have been studied by measuring the unsteady pressure on the floor of three cavities of different scales. The cavities have a lengthto-depth ratio of 5 and length-to-width ratio of 2 and the corresponding linear dimensions are in the ratio $0.5: 1: 2$. The experiments were conducted with clean cavities and cavities fitted with leading-edge sawtooth spoilers, so as to study the influence of scaling on clean cavities as well as the effectiveness of the passive control method on different sized cavities. The results showed significant variation of certain spectral characteristics of the clean cavities. The control effectiveness of the spoilers also showed variations with a change in scale of the model. It is recommended that before implementing a passive control device for practical applications, the device should be tested in the possible range of cavity length-toboundary layer thickness ratio $(\mathrm{L} / \delta)$ that can be experienced in actual flight.
\end{abstract}

\section{Nomenclature}

$\mathrm{C}_{\mathrm{p}} \quad=$ pressure coefficient $=\frac{2}{\gamma \mathrm{M}^{2}}\left[\frac{\mathrm{P}}{\mathrm{P}_{\infty}}-1\right]$

\footnotetext{
${ }^{1}$ PhD Researcher, Aeromechanical Systems Group, v.thangamani@cranfield.ac.uk

${ }^{2}$ Professor, Aeromechanical Systems Group, k.knowles@cranfield.ac.uk

${ }^{3}$ Senior Lecturer, Aeromechanical Systems Group, a.j.saddington@cranfield.ac.uk
} 


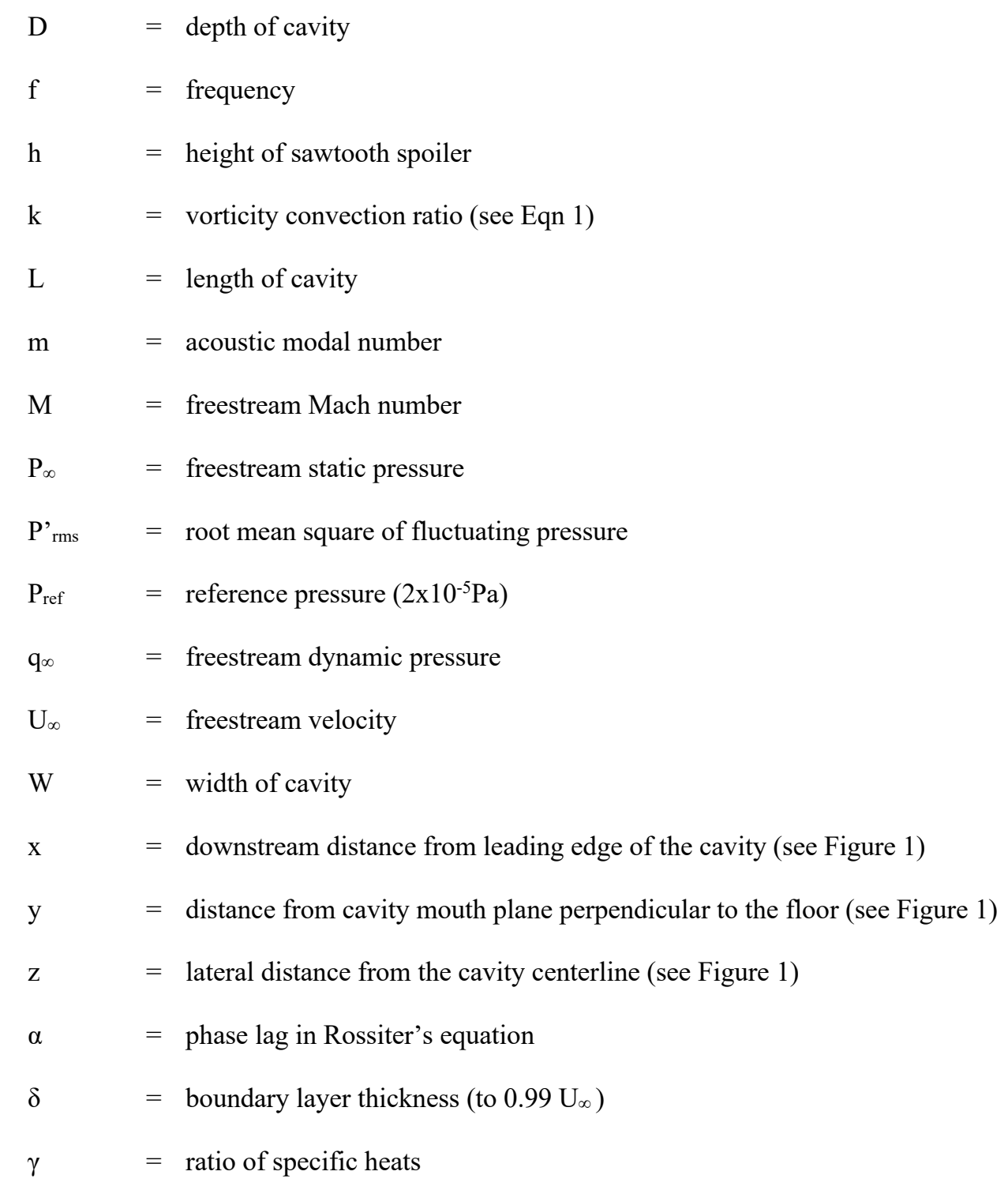

\section{Introduction}

$\mathrm{F}^{\text {LOW over certain cavity geometries can lead to intense pressure fluctuations inside and in the vicinity of the }}$ cavity and can lead to significant unsteady aerodynamic and acoustic loads on aircraft weapons bays, wheel wells and fuelling ports. Hence, suppression of cavity oscillations is of practical relevance and is particularly crucial in the case of a weapons bay. Significant aeroacoustic loads can cause damage to the aircraft components housed inside the weapons bay during the release of stores. Numerous techniques have been proposed to control the cavity oscillations and although some of them are very effective, it is not known whether they do so under all potential 
flight conditions. One variable that can significantly offset the performance of control devices, especially passive devices, is the Mach number. Some researchers have studied the effect of Mach number on their control devices and have found considerable variation in their performance [1-3]. Another crucial factor that can affect the performance of control devices is the scale of the model, whose influence is of significant practical importance $[4,5]$. The influence of the scale of the model can also be seen as a change in the thickness of the incoming boundary layer with respect to the model. This is a very important parameter without which a control technique cannot be successfully implemented on an actual aircraft weapons bay. Its relevance can be seen in two different aspects. First, at a given Mach number, cavities can be exposed to different thicknesses of the incoming boundary layer depending on factors such as the position of the weapons bay on the airframe and the altitude at which the aircraft is flying. Secondly, it determines the factors to be considered when a control technique that has been tested in the wind tunnel alone, is implemented on an actual aircraft. In this work, we attempt to study the effectiveness and variation in performance of a passive flow control technique with respect to the change in model scale, which is an area that has not been well understood yet. To study this, a leading-edge spoiler is employed which is a technique that has already proven to give good suppression results [1, 6-9]. The effectiveness of sawtooth spoilers was tested on three models having the same geometric ratios but exposed to different values of cavity-length-to-boundary-layer-thickness ratio (L/ $\delta)$. The cavities all have a length-to-depth ratio $(\mathrm{L} / \mathrm{D})=5$ and length-to-width ratio $(\mathrm{L} / \mathrm{W})=2$. Unsteady pressure measurements on the cavity floor have been carried out to study the flow oscillation in the clean cavities as well as in the cavities with leading-edge control.

\section{Experimental Details}

All the experimental studies were carried out in the transonic, closed-circuit, ejector-driven wind tunnel facility at the Defence Academy of the UK, which has a working section of length $364 \mathrm{~mm}$ and cross section $206 \mathrm{~mm}$ x 228 $\mathrm{mm}$. Three models have been studied in this work and their respective linear dimensions are in the ratio 0.5: 1:2 (Table 1). The cavities are fixed in a test rig which forms part of the side wall of the wind tunnel. Figure 1 shows the generic cavity co-ordinate system.

Sawtooth spoilers are used as the passive control device. The spoilers were made of $2 \mathrm{~mm}$-thick aluminium sheet and are composed of triangular elements. Spoilers of 3 different heights were used for the experiments and the 
values of their height, h, are $8 \mathrm{~mm}(\mathrm{~S} 1), 4 \mathrm{~mm}(\mathrm{~S} 2)$ and $2 \mathrm{~mm}(\mathrm{~S} 3)$ respectively and their geometric details are given in Table 2. The unsteady pressure measurements were made with an integrated pressure measurement device, the Scanivalve ZOC22B and data were collected from 27 pressure tappings made on the cavity floor. For a given cavity, nine pressure tappings were spaced linearly in the streamwise direction at a uniform distance of $\mathrm{x} / \mathrm{L}=0.1$ from each other. Three such rows are placed at planes $\mathrm{z} / \mathrm{W}=0,0.1625$ and 0.325 which were referred to as centre plane (CP), offset plane 1 (OP1) and offset plane $2(\mathrm{OP} 2)$ respectively (see Figure 1). Data obtained from the unsteady pressure measurements were used for constructing power spectra, spectrograms and overall sound pressure level (OASPL) plots. Due to the use of pneumatic tubing, a frequency response correction was applied to the pressure readings. The natural boundary layer developed over the tunnel wall was used for the studies. The boundary layer thickness, $\delta$, was found to be $7.7 \mathrm{~mm}$ and the Reynolds number per unit length was calculated as $13.14 \times 10^{6} \mathrm{~m}^{-1}$. All the three cavities, (i.e cavity A, cavity B and cavity C) were tested at a freestream Mach number of $\mathrm{M}=0.71$ and the corresponding $\mathrm{L} / \delta$ values were found to be $10.35,20.70$ and 41.40 respectively. Cavity A and cavity B were additionally tested at $\mathrm{M}=0.85$

Table 1. Details of cavity dimensions tested

\begin{tabular}{|c|c|c|c|c|c|}
\hline Cavity & Length, L (mm) & Width, W $(\mathrm{mm})$ & Depth, D (mm) & L/D & L/W \\
\hline A & 80 & 40 & 16 & 5 & 2 \\
\hline B & 160 & 80 & 32 & 5 & 2 \\
\hline C & 320 & 160 & 64 & 5 & 2 \\
\hline
\end{tabular}

The analog signal from the ZOC22B is acquired by a modular amplifier system, a DEWE-RACK, which has an A/D convertor. The signal is amplified, conditioned and digitized before being sent to the PC. A National Instruments DAQ card 6036E is used as the interface between the PC and DEWE-RACK. For the spectral analysis of the unsteady pressure samples, a total of 65536 samples averaged over four tunnel runs were recorded at a sampling rate of $12.5 \mathrm{kHz}$. The power spectra were plotted using an FFT algorithm, to determine the amplitude of 
the different frequency components. The experiments were found to be repeatable. The maximum error over four different runs was found to be $0.208 \mathrm{~dB}$ in tone amplitude and $1.93 \mathrm{~Hz}$ in tone frequencies. The experiments were also repeated on different occassions to test the repeatability. The same time series has been used in constructing the spectrograms. The spectrograms were constructed using a 512-point FFT with a temporal and frequency resolution of 20.5 milliseconds and $24.4 \mathrm{~Hz}$ respectively.

Table 2. Geometric details of different spoilers

\begin{tabular}{|c|c|c|c|c|c|}
\hline Spoiler & \multirow{2}{*}{$\begin{array}{c}\text { Height of triangular } \\
\text { element, } \mathrm{h}(\mathrm{mm})\end{array}$} & $\begin{array}{c}\text { Width of triangular } \\
\text { element }(\mathrm{mm})\end{array}$ & \multicolumn{3}{|c|}{ Number of teeth } \\
\cline { 4 - 6 } & 8 & 10 & Cavity A & Cavity B & Cavity C \\
\hline S1 & 8 & 20 & - & 8 & 16 \\
\hline S1a & 4 & 10 & - & 8 & 8 \\
\hline S2 & 2 & 5 & 8 & - & - \\
\hline S3 & & & & & - \\
\hline
\end{tabular}

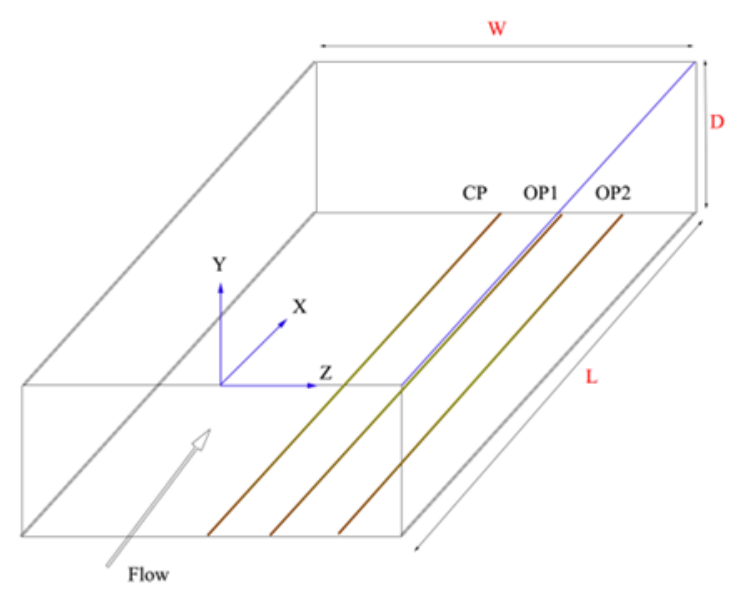

Figure 1. Cavity geometry and coordinate system 


\section{Results and Discussion}

\section{A. Spectral Analysis of Clean Cavities}

The oscillations in a cavity can be explained by studying the frequency spectrum of the unsteady pressure signal obtained from within the cavity. Figure 2 shows the narrowband spectra for the different clean cavities (cavities without spoilers) at $\mathrm{x} / \mathrm{L}=0.9$ (on the centre-line plane, $\mathrm{CP}$ ) for $\mathrm{M}=0.85$ and $\mathrm{M}=0.71$ plotted in terms of Strouhal numbers. It can be noticed that the spectra are constituted of broadband and multiple distinct 'peaks'. For cavity B, at $\mathrm{M}=0.85$, these peaks are formed at frequencies corresponding to $460.8 \mathrm{~Hz}, 1229 \mathrm{~Hz}$ and $1952 \mathrm{~Hz}$. These are known as the cavity tones. The feedback oscillation mechanism proposed by Rossiter [6] is suitable for explaining the physics of cavity flow dynamics in many situations. Cavity oscillation studies by various researchers have found good agreement of the peaks obtained by them with the modified Rossiter formula [10] and this is used as a technique to identify the Rossiter peaks. To find whether the oscillations here are caused by the mechanism explained by Rossiter, we evaluate the theoretical Rossiter frequencies using the empirical equation

$$
\frac{\mathrm{fL}}{\mathrm{U}_{\infty}}=\frac{\mathrm{m}-\alpha}{\mathrm{M}_{\infty}\left[1+\left(\frac{\gamma-1}{2}\right) \mathrm{M}_{\infty}{ }^{2}\right]^{-0.5}+1 / \mathrm{k}}
$$

where $\mathrm{f}$ is the frequency of the $\mathrm{m}^{\text {th }}$ mode, $\mathrm{m}=1,2,3 \ldots ., \alpha$ is a phase constant between the downstream propagating vortical disturbances and the upstream pressure waves and $1 / \mathrm{k}$ is a vorticity parameter. Based on a review of past literature, $\alpha$ and $\mathrm{k}$ have been taken as 0.31 and 0.57 respectively.This gives the values of frequencies for $\mathrm{M}=0.85$ and $\mathrm{L}=0.16 \mathrm{~m}$ as $464.9 \mathrm{~Hz}, 1138.8 \mathrm{~Hz}$ and $1812.7 \mathrm{~Hz}$ for $\mathrm{m}=1,2$ and 3. These are known as Rossiter frequencies and correspond to the different modes of cavity oscillation. By comparing the calculated values with the peaks obtained in the frequency spectrum, it can be seen that there is a reasonably good agreement between the values. The difference between experimental and theoretical values are within the bounds reported by Ahuja and Mendoza in their extensive benchmark study of numerous cavity dimensions [4]. They noticed that experimental values lie within an average of $20 \%$ of the theoretical values and also observed a larger deviation in cavity tones from 
Rossiter's prediction at lower Mach numbers. Therefore these peaks can be called the Rossiter tones and hereafter, they will be denoted as R1, R2 and R3.

From the spectrum, it can be observed that there are other peaks besides the ones described by Rossiter's equation. These peaks can arise due to the interaction between different Rossiter modes and their harmonics and sub-harmonics. The different frequencies interact non-linearly with each other and the resultant frequency is the sum/difference/multiple or a combination of the constituent frequencies [11] .

The spectra measured at different points on the cavity floor had peaks at the same frequencies which show that the cavity oscillations are global in nature. For the spectra for cavity B at $\mathrm{M}=0.71$ (see Figure $2 \mathrm{~b}$ ), the peak frequencies measured are $498.2 \mathrm{~Hz}, 1072 \mathrm{~Hz}$ and $1646 \mathrm{~Hz}$ and the corresponding values according to the modified Rossiter's formula are $413.33 \mathrm{~Hz}, 1012 \mathrm{~Hz}$ and $1611.4 \mathrm{~Hz}$ respectively. It can be seen that while the second and third modes are well predicted by the equation, the first mode is under-predicted in this particular case. It can also be observed that the dominant tone here is R2 $(1072 \mathrm{~Hz})$ and has very high amplitude compared to the other tones $(\mathrm{SPL}=161.3 \mathrm{~dB})$.

For cavity $\mathrm{C}$ at $\mathrm{x} / \mathrm{L}=0.9$ for $\mathrm{M}=0.71$ (Figure $2 \mathrm{~b}$ ), all the salient features explained for cavity $\mathrm{B}$ can be observed here as well. The Rossiter tones R1, R2 and R3 occur at $206.8 \mathrm{~Hz}, 553.9 \mathrm{~Hz}$ and $946 \mathrm{~Hz}$ respectively. In this case also, the dominant mode is R2 $(553.9 \mathrm{~Hz})$. Near R1, there are a range of frequencies with high amplitude. The deviation for R1 of cavity B and R3 of cavity C from modified Rossiter's formula is $20.4 \%$ and $17.4 \%$ respectively just at the limit of Ahuja and Mendoza's observed accuracy for the Rossiter model [4]. High amplitude tones observed in a cavity can be due to Rossiter's mechanism, longitudinal oscillations ("duct oscillations") or through a non-linear interaction of the different modes and their harmonics or sub-harmonics. Bispectral analysis carried out on the current cases indicated that R1 of cavity B and R3 of cavity C were self-excited and were not formed due to non-linear interaction of other tones. The duct oscillation frequencies of cavities $\mathrm{B}$ and $\mathrm{C}$ were very close to the second mode of oscillation (R2) of the cavities. Hence it is probable that the peaks R1 of cavity B and R3 of cavity $\mathrm{C}$ are due to Rossiter's mechanism.

Unlike cavity B and cavity $\mathrm{C}$, the peaks obtained by plotting the spectra of cavity A are not prominent for $\mathrm{M}=$ 0.71 (Figure 2b). R1, R2 and R3 occur at $972.7 \mathrm{~Hz}, 2373 \mathrm{~Hz}$ and $3885 \mathrm{~Hz}$ for $\mathrm{M}=0.85$ and $836.9 \mathrm{~Hz}, 2061 \mathrm{~Hz}$ and 
$3454 \mathrm{~Hz}$ for $\mathrm{M}=0.71$. The Strouhal numbers for $\mathrm{M}=0.71$ appear to be $0.28,0.69$ and 1.13 as against the theoretical values of $0.28,0.69$ and 1.10 corresponding to $\mathrm{m}=1,2$ and 3 . However, the Rossiter tones become more prominent for cavity A when the Mach number is increased to 0.85 , as seen in Fig 2a. R1 is the dominant tone for Cavity A at both the Mach numbers.

Figure 3 shows the measured tone Strouhal numbers compared with the theoretical predictions by Rossiter and Heller \& Bliss [10]. Except R1 for cavity B (at $\mathrm{M}=0.71)$ and R3 for cavity $\mathrm{C}($ at $\mathrm{M}=0.71)$, all other values agree well and fall within $10 \%$ of the theoretical predictions. The scatter in data of Strouhal numbers was observed by various researchers $[4,12-14]$. The deviation from theoretical values arises from the choice of the constants $\alpha$ and $\mathrm{k}$ in Rossiter's equation. Though researchers use empirical values based on data-fit, these values depend on various conditions including the incoming boundary layer characteristics. As mentioned before, the two quantities with maximum deviation occurred for cavity $\mathrm{B}$ and cavity $\mathrm{C}$ at $\mathrm{M}=0.71$. It is established from the discussions that the dominant frequencies of oscillation in all the cavities are created by the Rossiter feedback mechanism and the aim of the passive control technique is to disrupt the feedback loop.

It can be seen from Figure 3 that there is no significant shift in the measured Strouhal numbers of the corresponding modes of the different cavities and all the values, except the two above-mentioned cases, lie within an absolute deviation of $10 \%$ from the theoretical values. This shows the validity of Rossiter's feedback formula across different scales. It can also be noticed that the amplitude of oscillation modes along with the broadband levels increase with increase in $\mathrm{L} / \delta$ (Figure 2). One possible explanation for this behaviour can be given using the cavity oscillation model proposed by Heller \& Bliss [15]. According to their model, the unsteady motion of the shear layer causes a periodic mass addition and removal process at the cavity trailing edge. This process produces a piston-like effect at the rear bulkhead, which sets up an internal wave structure that forces the shear layer. This shear layer motion is responsible for the trailing-edge mass addition and removal. When a shear layer is disturbed by an acoustic wave, the disturbance amplifies in the direction of the flow and the shear layer displacement becomes a maximum at the trailing edge. With an increase in length, this amplification of the shear layer increases and so does the displacement of the shear layer from the mean position at the trailing edge. This leads to an increase in the 
amplitude of pressure pulses produced by the 'pseudopiston' and is responsible for the high sound pressure levels observed in cavity $\mathrm{C}$.

\section{B. Time-Frequency Analysis of Clean Cavities}

It has been shown from the results of the frequency spectra (Figure 2) that multiple modes of oscillation exist in all the cavities with a very visible dominant mode of oscillation noted for cavities B and C. Oscillation of the shear layer in multiple modes leads to another critical question - whether these modes coexist all the time or if there is a 'mode switching' occurring between them. Kegerise et al. [11] suggested that the Rossiter-mode peaks in an unsteady pressure spectrum may not coexist independently of each other but might be a result of mode switching. Experimental studies in reference [11] at $\mathrm{M}=0.6$ for a cavity of $\mathrm{L} / \mathrm{D}=4$ showed the existence of mode switching and the dominant mode was seen to switch between primary Rossiter modes. The mode-switching phenomenon has also been numerically investigated by Gloerfelt et al. [16]. The dominant mode at a particular instant corresponds to the number of vortices in the shear layer bridging across the cavity.

Since the frequency spectrum does not give information about the temporal behaviour of the different modes, we employ time-frequency analysis to study the same. Figure 4 shows the spectrogram of cavity C. It can be seen that the second mode, R2, is the only mode that is present for the major part of the measurement time. Its strength is almost the same, barring mild fluctuations, for most of the time except for the two intervals : $0.3277-0.3482 \mathrm{~s}$ and $0.7782-0.8192 \mathrm{~s}$. In these two intervals the second mode is almost non-existent. In contrast, the third mode, R3, can be observed to be energetic only during these two periods. This is significant and clear evidence of mode switching between R2 and R3. During these periods, the energy of the flow is extracted by the cavity oscillation in the third mode and accounts for the high amplitude observed for R3 in the power spectrum of cavity C. Similar to the observation of previous researchers, here also the mode switching seems to occur randomly and not periodically throughout the sampling. However, longer time samples are required to verify this. The presence of R1 is not consistent with time and occurs randomly and mostly coexists with R2. From the above observations, it follows that any control experiment for cavity $\mathrm{C}$ should be designed to suppress $\mathrm{R} 2$ since it is the most dominant tone in terms of amplitude as well as prevalence in the time domain. This aspect becomes very pertinent in active control systems which are designed for forcing the shear layer at a particular frequency. 
The evidence of mode switching was observed for cavity $\mathrm{B}$ as well at $\mathrm{M}=0.71$. In this case, there is a mode switching occuring between R2 and R1. It can be seen that R2 has its presence felt over most of the time period except intervals like $0.3277-0.3891 \mathrm{~s}$ and $0.6758-0.7782 \mathrm{~s}$ where it is dormant (Figure 5). At this interval R1 can be seen to dominate the spectrum. The occurrence of R1 during the other intervals is not prominent due to the presence of R2. The presence of R3 is intermittent and feeble during most of the time intervals. For $\mathrm{M}=0.85$, there was no conspicuous presence of mode switching and R1 coexisted for a major part of the measurement time (from 0 $-0.8806 \mathrm{~s}$ ) with R2. From the literature, mode switching has not been reported in all cases and the reason behind the occurrence of mode switching or coexistence of modes is still not clear.

For cavity A, because the peaks are less distinct than for cavities B and C, it is difficult to identify the time trace of R1 amidst the heavy envelope of broadband frequencies. Nevertheless, R2 and R3 are distinct and show some levels of intermittency. The occurrence of R2 seems to be periodic with strong presence during the intervals 0.2867 $0.4915 \mathrm{~s}, 0.5939-0.7968 \mathrm{~s}, 0.7987-0.9011 \mathrm{~s}$ and $0.9626-1.065 \mathrm{~s}$.

Time-frequency analysis of all the cases shows that mode-switching occurs for cavities B and C for $\mathrm{M}=0.71$. When mode switching occurred, the dominant frequency switched from one mode to another. Also a larger deviation from Rossiter's formula was observed in the frequency of the new mode. The reason behind the large deviation might not be the phenomenon of mode switching but due to the change in flow conditions between the switches. Such changes are not represented by Rossiter's semi-empirical formula. The deviation in the frequency values from Rossiter's equation when such mode switching occurs has not been reported before. No such deviation was observed in other cases when mode switching was absent. During the mode switching, the energy was almost completely redirected from one mode to another. Gloerfelt et al. [16] note that mode switching is more likely to be observed in fully turbulent shear layers i.e. at high Reynolds numbers. The vortices are then clusters of small scales rather than a single roll. It was also observed that the dominant frequency's presence was felt for more than $90 \%$ of the measurement time. The main aim of the control experiments would be to suppress the broadband as well as these dominant tones which follow Rossiter's mechanism. This is done by disrupting the feedback mechanism at one or more critical points. 


\section{Mode Shapes}

From the spectrogram studies it was shown that all the primary modes did not always coexist and there was mode-switching occurring between them. While certain modes were relatively 'steady' with time and existed for longer periods of the measurement, the occurrence of certain other modes was random. For example, R3 of cavity C (at $\mathrm{M}=0.71$ ) was found to be dominant only for two short intervals i.e. $0.3277-0.3482 \mathrm{~s}$ and $0.7782-0.8192 \mathrm{~s}$ which is approximately 19 cycles and 38 cycles respectively (although these cycle lengths are not thought to be significant, as other examples were seen on other cycle lengths). However R2 occurred for more than 95\% of the measurement time and it would be useful to look at the mode shape of such dominant tones for the different cavities. Figures 7 and 8 show the variation of the SPL values of the dominant tones of different cavities and their corresponding theoretical mode shapes that have been developed by Smith \& Shaw [17]. The equation as a function of modal number $(\mathrm{m})$, cavity $\mathrm{L} / \mathrm{D}$ and streamwise position $\mathrm{x} / \mathrm{L}$ can be written as

$$
(\mathrm{SPL})_{\overline{\mathrm{L}}}=(\mathrm{SPL})_{\frac{\mathrm{x}}{\mathrm{L}}=1}-10\left[1-\left|\cos \left(\pi \mathrm{m} \frac{\mathrm{x}}{\mathrm{L}}\right)\right|+\left(0.33\left(\frac{\mathrm{L}}{\mathrm{D}}\right)-0.6\right)\left(1-\frac{\mathrm{x}}{\mathrm{L}}\right)\right]
$$

The value at $\mathrm{x} / \mathrm{L}=1$ was calculated based on the value obtained at $\mathrm{x} / \mathrm{L}=0.9$. It can be seen from Figures 7 and 8 that the measured mode shapes are in general agreement with the theoretical mode shapes. Mode shapes corresponding to different mode numbers, undergo different phase changes within the cavity. The mode R1 undergoes a phase change of $\pi$ across the cavity length. It can be observed that there is a small lag of the measured shapes from the theoretical mode shapes. This slight variation is due to the assumption made for the equation that the pressure anti-node occurs at the rear wall while in reality it occurs slightly upstream of the rear wall. This is a manifestation of the periodic mass addition and removal at the trailing edge. Further, the accuracy of the mode shapes is also limited by the spatial resolution of the measurements made.

\section{Cavity with Passive Control}

Pressure measurements in the cavities were repeated with the spoilers fitted at the leading edge. Spoilers of three different heights have been used in the experiments, namely $\mathrm{S} 1(\mathrm{~h} / \delta=1.035), \mathrm{S} 2(\mathrm{~h} / \delta=0.517)$ and $\mathrm{S} 3(\mathrm{~h} / \delta=0.258)$. 
An additional spoiler S1a, which has the same value of $\mathrm{h} / \delta$ as $\mathrm{S} 1$ but half the number of teeth was also used for cavity C (see Table 2).

Figure 9 shows the centreline mean pressure distribution on the cavity floor with the use of various spoilers. It can be seen that with the use of spoilers, there is significant reduction of pressure on the cavity floor. The pressure reduction increases with increase in the spoiler height. This indicates a possible lifting of the shear layer by the spoiler. Experimental and numerical studies with fences and cylindrical rods placed on the cavity leading edge show a similar observation [3]. The presence of the spoiler in front of the cavity causes the flow in front of it to decelerate and create a region of high pressure. This causes the shear layer to deflect towards the freestream instead of going into the cavity. This 'lifting' of the shear layer can potentially affect the interaction of the shear layer with the aft wall and reduce the feedback strength which leads to a decrease in the oscillation amplitude. With the increase in spoiler height, the deflection angle is increased, which further reduces the pressure levels and feedback intensity within the cavity. The decrease in pressure from S2 to S1 is more than the decrease in pressure from S3 to S1. Also, there is a pressure rise in all the cases near the trailing edge, indicating a weak interaction between the shear layer and aft wall. The extent of the mean pressure reduction, however, does not correlate directly with the unsteadiness in the cavity. The mean pressure distribution indicates a change in the mean flow structures and does not necessarily reflect any disruption of the feedback cycle.

To find the effect of spoilers on the unsteadiness of the cavity, the power spectra of the cavity are plotted. From Figures 10 and 11 it can be seen that, with the use of spoilers, there is a decrease in both the broadband as well as tonal amplitudes for cavities A and B for both Mach numbers. The only exception is R1 of cavity B at M $=0.71$, which shows a slight increase from the clean cavity with the use of S2. The decrease in the unsteadiness increases with the height of the spoiler. For $\mathrm{M}=0.71$, cavity $\mathrm{A}$ has a maximum reduction of $5 \mathrm{~dB}$ in the dominant tone (R1), while for cavity $\mathrm{B}$ there is a maximum reduction of $12.7 \mathrm{~dB}$ for the dominant tone (R2), which is very high. For $\mathrm{M}=$ 0.85 , there is a reduction of $5.7 \mathrm{~dB}$ for cavity $\mathrm{A}$ in the dominant tone, whereas there is a maximum reduction of 11.4 $\mathrm{dB}$ for cavity $\mathrm{B}$ in the dominant tone. However, these are only tonal attenuations and to assess an overall reduction in the total energy of the all the frequencies, Overall Sound Pressure Levels (OASPL) have to be studied, which will be discussed later. For cavities A and B, it can be observed that the frequency spectra are almost reduced to 
broadband . This shows a significant disruption of the feedback mechanism by the spoilers leading to a complete attenuation of cavity tones. For cavity $\mathrm{C}$ there is less reduction in the cavity unsteadiness. Although there is a reduction of $7.2 \mathrm{~dB}$ in the dominant tone, the power spectrum is not reduced to mere broadband. There are peaks present even after the use of spoilers, although there is a reduction in their amplitude. The reduction is slightly increased when a spoiler with the same $\mathrm{h} / \delta$ but fewer teeth (S1a) is used. The results for cavity $\mathrm{C}$ prove the loss in the effectiveness of spoilers as a tone suppression method for large values of $L / \delta$. Another observation that can be noted from the figures is the increase in the tonal frequencies when the spoilers are used. Cavities A, B and C show respectively $24.1 \%, 24.1 \%$ and $29.2 \%$ of increase in the value of R2. This increase in frequency may be caused by an increase in convective velocities of vortices in the deflected shear layer and/or an increase in the reverse velocities inside the cavity with the use of spoilers.

Investigating the exact mechanism of suppression is crucial for better design of the control techniques and improving the parameters involved. Although the mechanisms cannot be explained without flow visualization, a plausible explanation can be inferred from the literature review and pressure measurements that have been conducted. Mean pressure distributions indicate a lifting of the shear layer away from the cavity into the freestream. This lifting of the shear layer causes its reattachment to be shifted further downstream. In effect, this reduces the mass addition into the cavity as explained before using the model of Heller \& Bliss [15]. The amount of mass addition is proportional to the mean magnitude of the velocity vector normal to the aft wall. When the shear layer impinges at the aft wall at a deeper point, the magnitude of velocity is expected to be higher. But when the shear layer is shifted away from the aft wall, the magnitude becomes less. In the numerical simulations by Ashworth [8], the shear layer continued to rise steadily for $1 / 5^{\text {th }}$ of the cavity length with the use of a spoiler. When $L / \delta$ increases, the shear layer is allowed sufficient length to get enough amplification to enable mass addition, and consequently pressure oscillations, at the trailing edge. This manages to initiate the Rossiter feedback mechanism and could be responsible for the failue of spoilers to attenuate the tones completely in a large scale cavity.

The attenuation of tones observed for cavities A and B could also be due to the reduction of the spatial coherence of the shear layer by the spoilers which in turn reduces the strength of the Kelvin-Helmholtz instability waves that are responsible for the shear layer disturbances. The shear layer also becomes thickened with the use of 
spoilers, which results in the reduction of cavity unsteadiness. A thicker shear layer with smoother velocity gradients is less responsive to the pressure disturbances.

Whilst the reason for using spoilers is their tone attenuation properties, it is recognized that their drag may be an issue for aircraft applications, albeit that spoilers will only be deployed transiently. This was not something that was investigaterd in the present study and would have required pressure tappings on the spoilers and on the cavity rear wall. We may speculate, however, that while the spoilers produce resistance to the freestream flow, the drag produced on the aft-wall of the cavity is likely to be reduced when the shear layer is deflected away from it. Hence it is possible that the two phenomena may balance and the overall drag change for weapons bays might not be significant. The load on the spoilers, however, may be of concern.

\section{E. OASPL Suppression}

To assess the effectiveness of a control technique, it is not sufficient to study the effect of the technique on tones alone. The impact on the total energy of the spectra is also an important consideration in the design of a control technique. The Overall Sound Pressure Level (OASPL) gives the total energy under the frequency spectrum and describes the contribution of all the frequencies. It is expressed as

$$
\text { OASPL }=20 \log \left(\frac{\mathrm{P}^{\prime} \mathrm{rms}}{\mathrm{P}_{\mathrm{ref}}}\right)
$$

where $\mathrm{P}^{\prime}$ rms is the root mean square of the fluctuating pressure and $\mathrm{P}_{\text {ref }}$ is the international standard for the minimum audible sound, which has the value of $2 \times 10^{-5} \mathrm{~Pa}$.

Figure 12a shows the OASPL distribution for the three cavities at $\mathrm{M}=0.71$. It can be seen that the OASPL generally has higher values near the trailing edge. This increase is due to the amplification of the shear layer disturbances in the downstream direction. For cavity A, the OASPL rises monotonically downstream of $50 \%$ of the cavity length. In the case of cavities B and C, the region of monotonically-increasing OASPL in the rear of the cavity becomes progressively shorter. The shapes of the OASPL curves have a variation similar to the mode shapes of the dominant tones of the respective cavities. This shows that the major contribution to the OASPL levels of the 
cavities is from the Rossiter tones. For cavities B and C, R2 is the dominant tone and the OASPL curve follows a variation similar to that of the amplitude of R2 along the streamwise direction. The steady increase in OASPL for cavity A from $\mathrm{x} / \mathrm{L}=0.5$ to $\mathrm{x} / \mathrm{L}=0.9$ is due to $\mathrm{R} 1$ being the dominant tone which has a steady rise in that range. For cavity A, the similarity of the OASPL curve to the mode shape of R1 is more evident in Figure $12 \mathrm{~b}$ for $\mathrm{M}=0.85$, where the Rossiter tones were more prominent. The OASPL values increase as the cavity size is increased, with a fixed boundary layer thickness.

The OASPL values for the cavities fitted with spoilers are calculated in the same way and Figure 13a shows the reduction in OASPL for the 3 cavities. It can be seen that the reduction levels obtained are very different for the three cavities and have a significant streamwise variation. Cavity $\mathrm{C}$ shows the best suppression for the front $60 \%$ of its length. It is followed by cavity B and then by cavity A. The decrease in suppression level as noted in this observation is due to the dominance of broadband turbulence in the frequency spectrum of cavity A. As the shear layer is close to the floor, the turbulence in the shear layer is felt by the cavity floor. The use of a spoiler at the leading edge increases the turbulence in the shear layer and thickens it. The increase in OASPL over much of the first $50 \%$ of the length of cavity $\mathrm{A}$ is due to the increase in broadband turbulence associated with this increase in shear layer turbulence. Similar observations have been made by Ukeiley and Ponton, in their work with a solid fence [7]. They found that sources in the shear layer contribute to the surface pressure sensed in the front part of the cavity so that not all of the surface pressure fluctuations are entirely due to the interaction between the shear layer and the aft wall. For the rear $20 \%$ of the length, cavity B shows the greatest reduction. The spoiler effectively reduces the feedback strength of the Rossiter mechanism but increases the turbulence of the shear layer. The influence of this increase in shear layer turbulence becomes significant with decreasing $L / \delta$. Cavity $C$ shows a reduction level similar to the OASPL variation across the cavity length because of the negligible effect of shear layer turbulence on the frequency spectrum of the cavity floor. Here the reduction is proportional to the reduction in the feedback strength and is not influenced by broadband turbulence and hence follows the shape of the OASPL variation. In general, it can be deduced that for a cavity with strong Rossiter tones, the variation of OASPL reduction in the streamwise direction is related to the variation of the amplitude of the dominant tone. With lower values of $\mathrm{L} / \delta$, this trend gets disturbed by the turbulence in the shear layer. 
The suppression levels show significant spanwise variation too. The variation of OASPL reductions at the three streamwise planes CP, OP1 and OP2 can be seen in the Figures 14-16. For both cavity A and cavity B, the reduction at $\mathrm{CP}$ and $\mathrm{OP} 2$ is out of phase with reduction at OP1. This observation is significant and shows that the use of a sawtooth spoiler significantly disturbs the coherence of the shear layer. The phase differences seen in the reductions correspond to the peaks and valleys in the sawtooth spoiler and prove that the shear layer impinges at different points along the span near the trailing edge. This causes the disturbance near the trailing edge to have phase differences which can be seen in the plots. This observation is clearer in Figure 15 for cavity B than cavity A, which is dominated by random broadband turbulence. This again corroborates the significance of shear layer turbulence on the cavity floor. As explained before, the shear layer influence is maximum on plane CP. The spoilers induce significant spanwise and streamwise three-dimensional effects in the shear layer. For cavity C the OASPL variation with the use of spoilers does not cause any significant spanwise variation, which implies a more coherent and less disturbed shear layer. This is partly responsible for the high-amplitude tones observed in the spectrum of cavity C.

The maximum suppression that has been measured is for cavity $\mathrm{C}$, which showed an $8.7 \mathrm{~dB}$ reduction at $\mathrm{x} / \mathrm{L}=$ 0.5. Figure $13 \mathrm{~b}$ shows the reductions for cavities $\mathrm{A}$ and $\mathrm{B}$ for $\mathrm{M}=0.85$. It can be seen that the trends of reductions are similar to the measurements made at $\mathrm{M}=0.71$ for cavity $\mathrm{B}$, though the suppression levels are higher. The reduction values are lower for cavity A for the front $60 \%$ of the cavity length. This observation shows that the disruption of the feedback loop increases at $\mathrm{M}=0.85$ for cavity $\mathrm{B}$, compared with $\mathrm{M}=0.71$. This could probably be due to increased lifting of the shear layer at $\mathrm{M}=0.85$ as the pressure values on the cavity floor were found to be lower in this case. The streamwise variation of OASPL reductions for cavity A shows a clearer trend at M $=0.85$ and is less influenced by the shear layer turbulence. The shape of the reduction curve is similar to the mode shape of the dominant tone R1 of cavity A (Figures 8 and 13b). This again corroborates the earlier statement that the reduction at a particular point is related to the amplitude of the dominant tone at that point.

OASPL reductions for the different cavities at constant $\mathrm{h} / \mathrm{D}$ shows that the effectiveness of a spoiler is also driven by the factor $\mathrm{h} / \delta$ (see Figure 17). The suppression levels are maximum for cavity $\mathrm{C}$ which has the largest value of $\mathrm{h} / \delta$ and minimum for cavity A which has the lowest value of $\mathrm{h} / \delta$. Rossiter found an increase in toneamplitude suppression with increase in $\mathrm{h} / \delta$ for a given cavity [6]. However, the findings here show that both $\mathrm{h} / \delta$ as 
well as $\mathrm{L} / \delta$ play a significant role in the performance of a leading-edge passive control device. It has been shown that the geometry of the spoiler as well as the cavity with respect to the incoming boundary layer are important parameters while designing a leading-edge passive control device. 


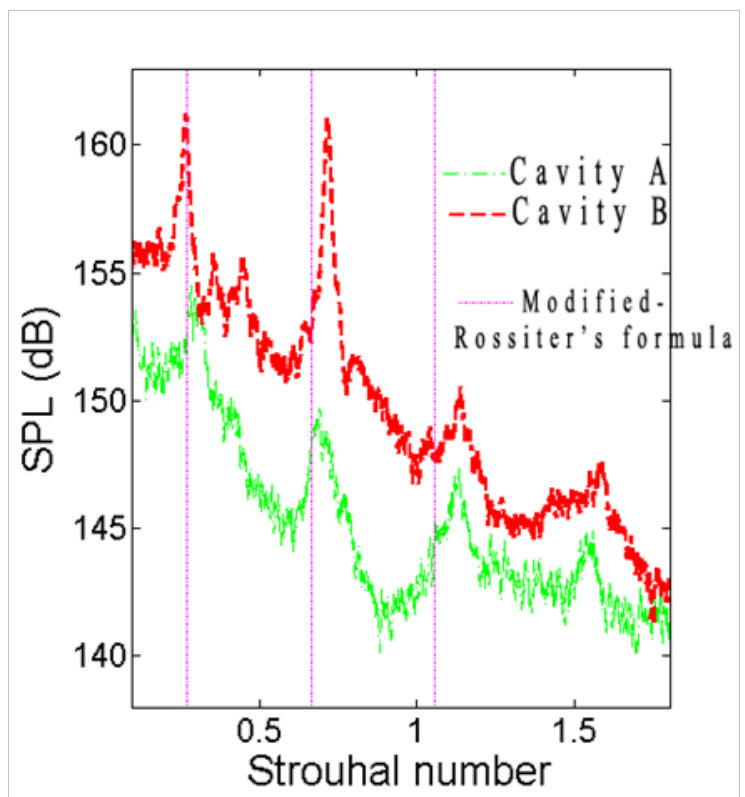

(a)

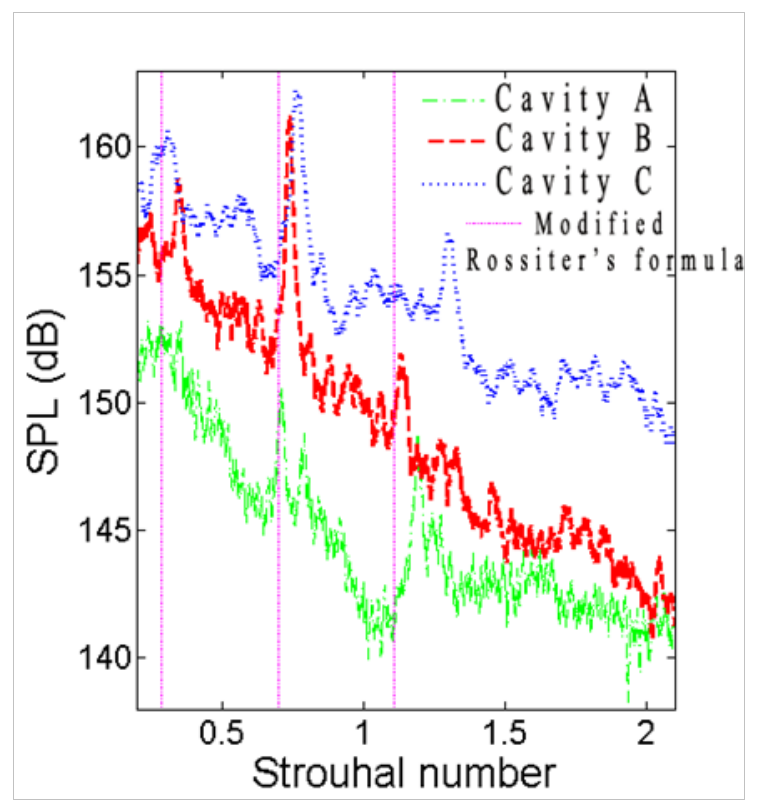

(b)

Figure 2. Pressure spectra at $x / L=0.9$ for different cavities at (a) $M=0.85$ and (b) $M=0.71$

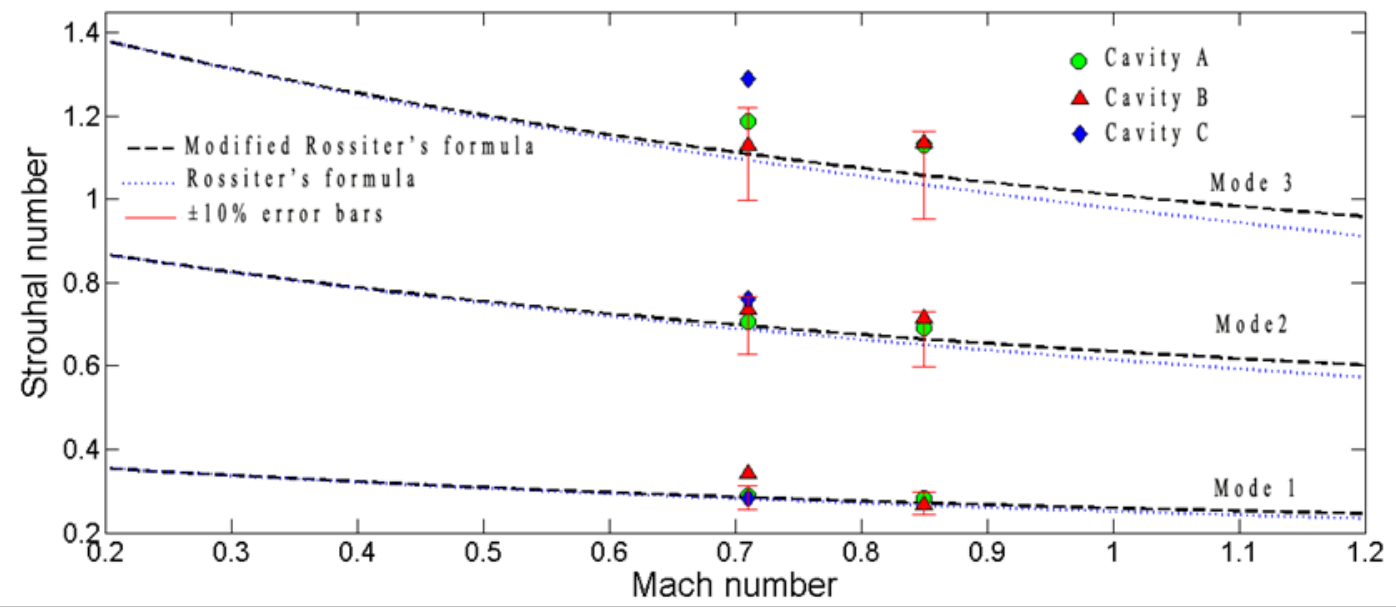

Figure 3. Strouhal numbers of different frequency modes $(M=0.71)$

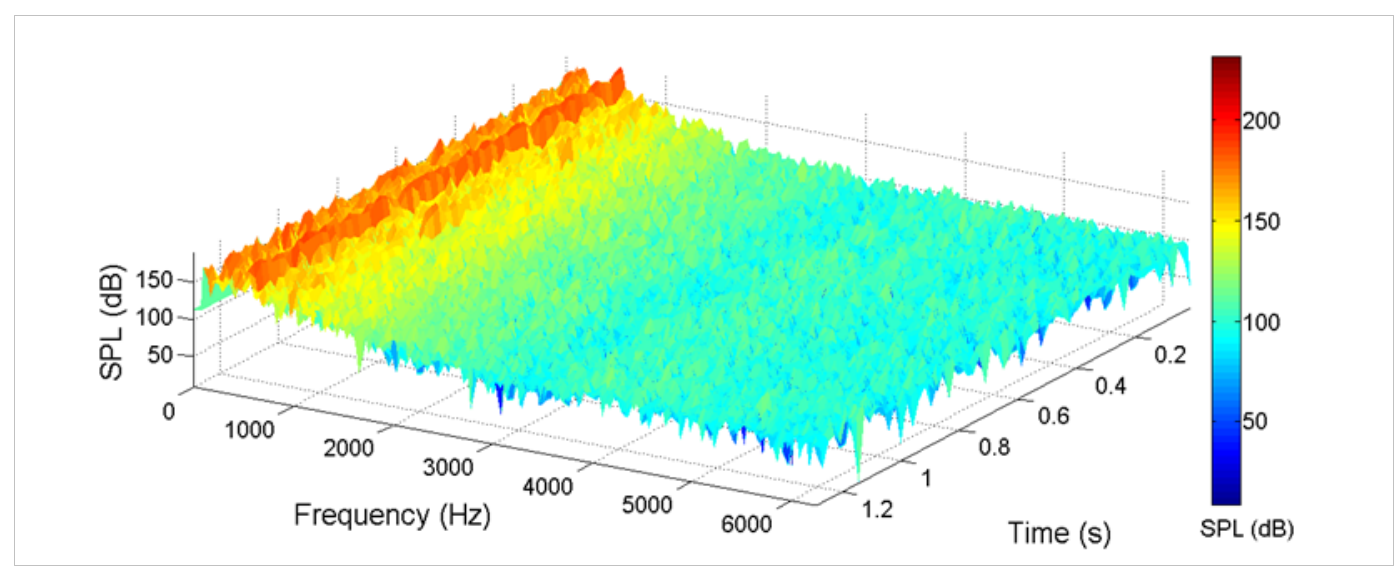

(a) 


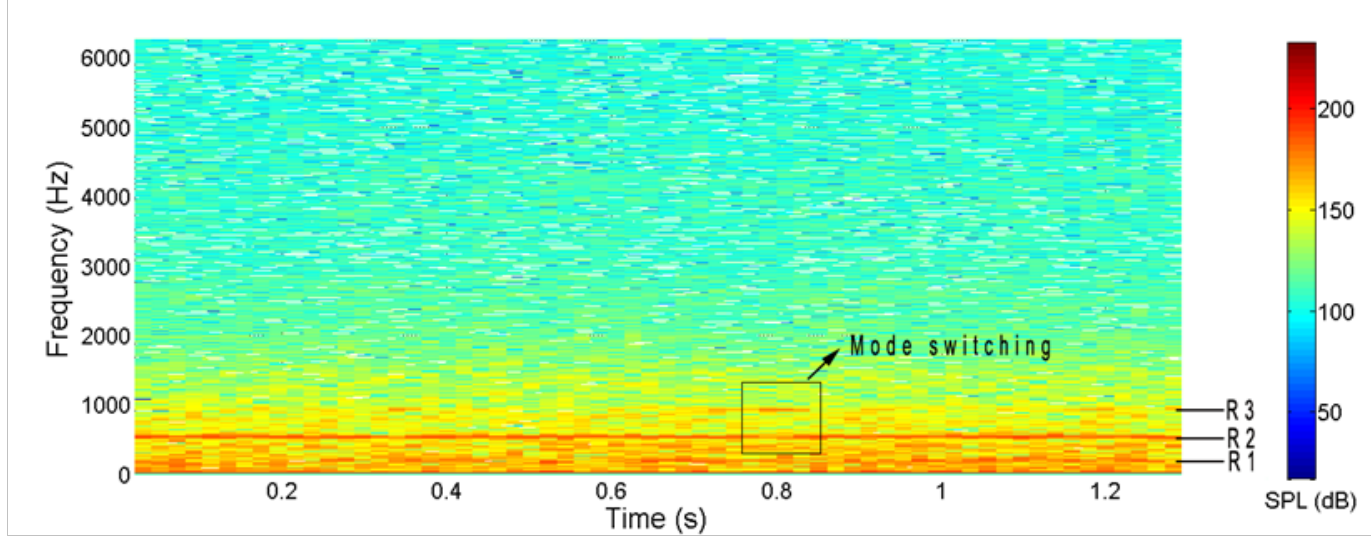

(b)

Figure 4. Spectrogram for cavity $C$ at $x / L=0.9$ at $M=0.71$ : (a) 3d and (b) plane view

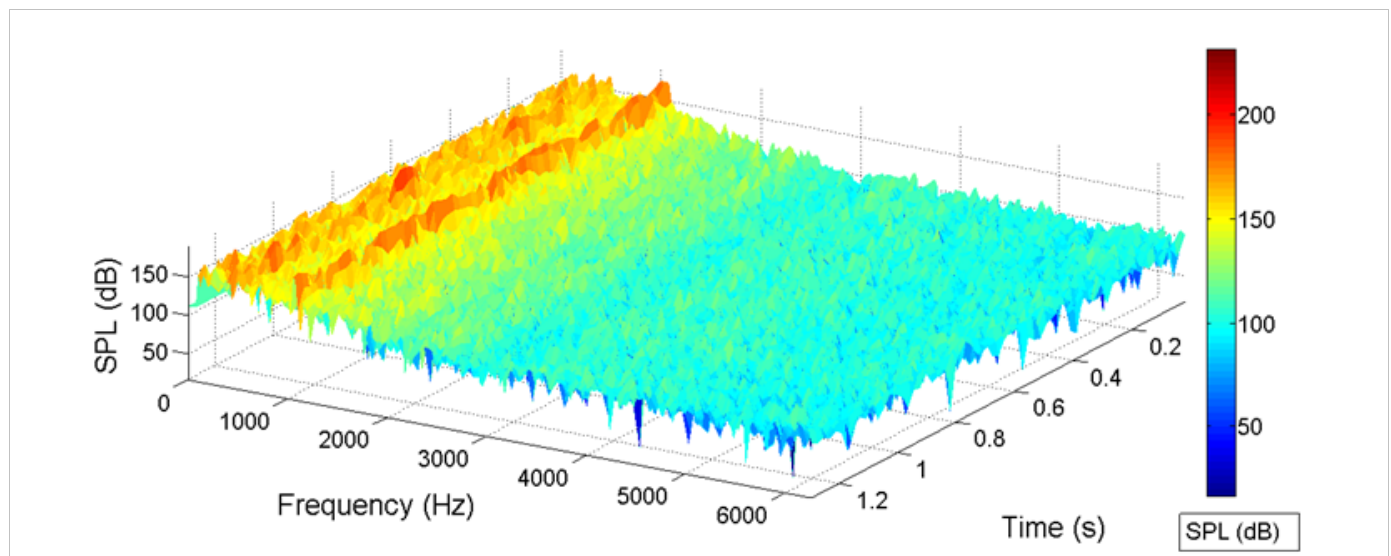

(a)

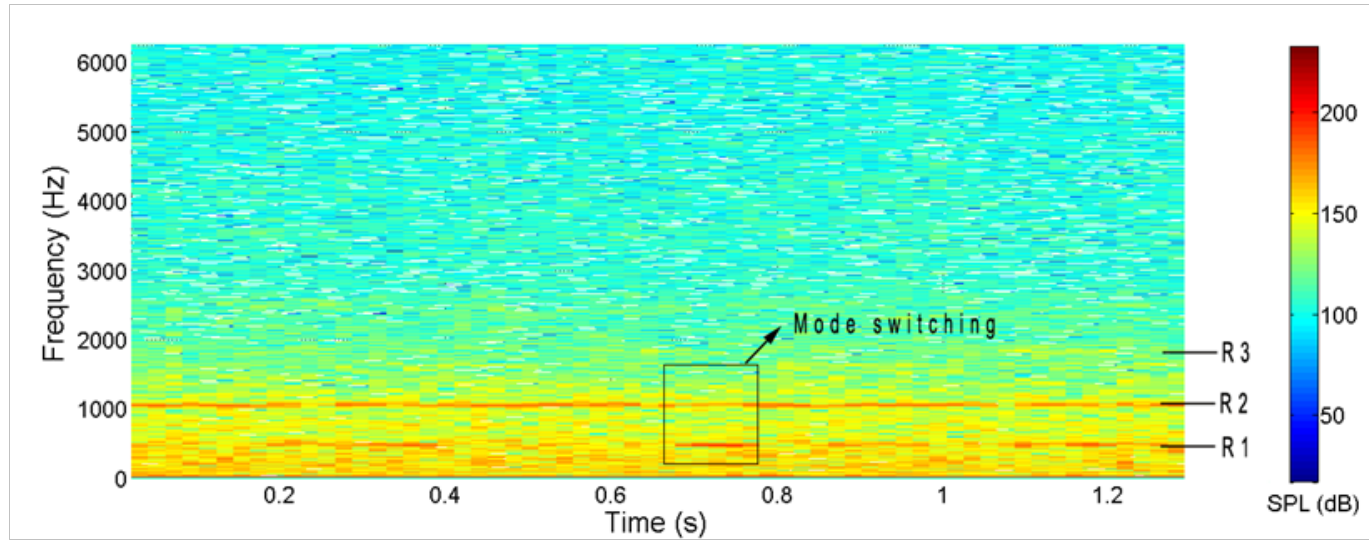

(b)

Figure 5. Spectrogram for cavity $B$ at $x / L=0.9$ at $M=0.71$ : (a) $3 d$ and (b) plane view 


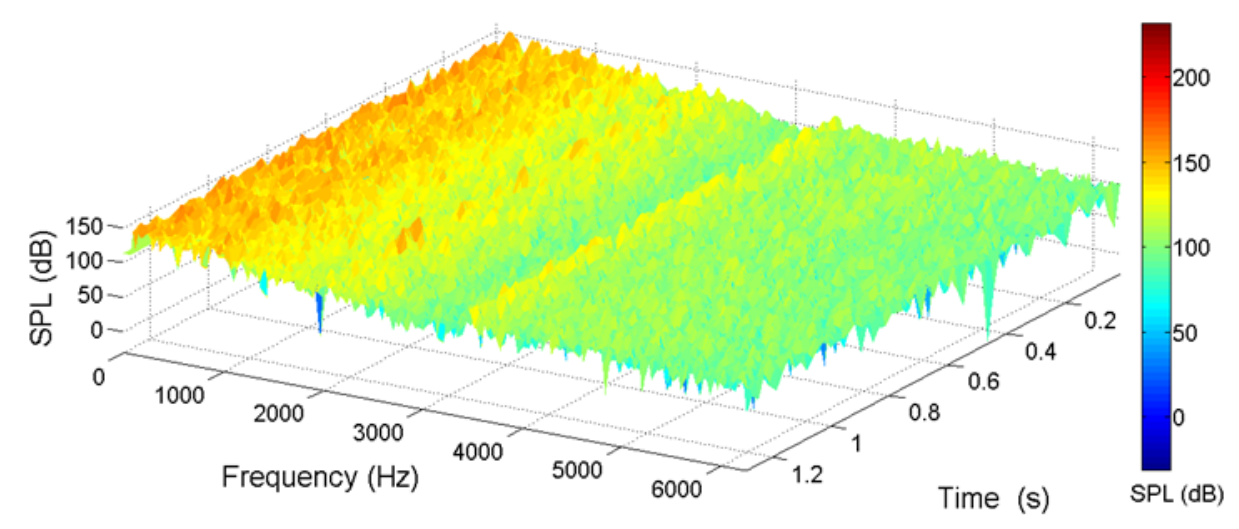

(a)

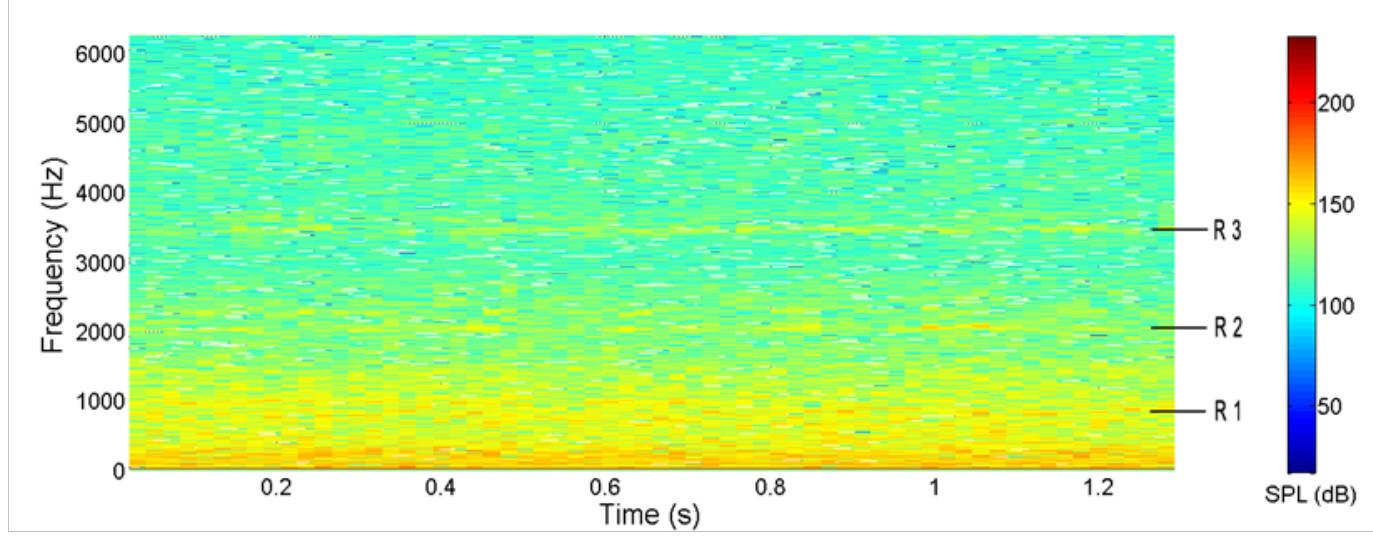

(b)

Figure 6. Spectrogram for cavity $A$ at $x / L=0.9(M=0.71):$ (a) $3 d$ and (b) plane view

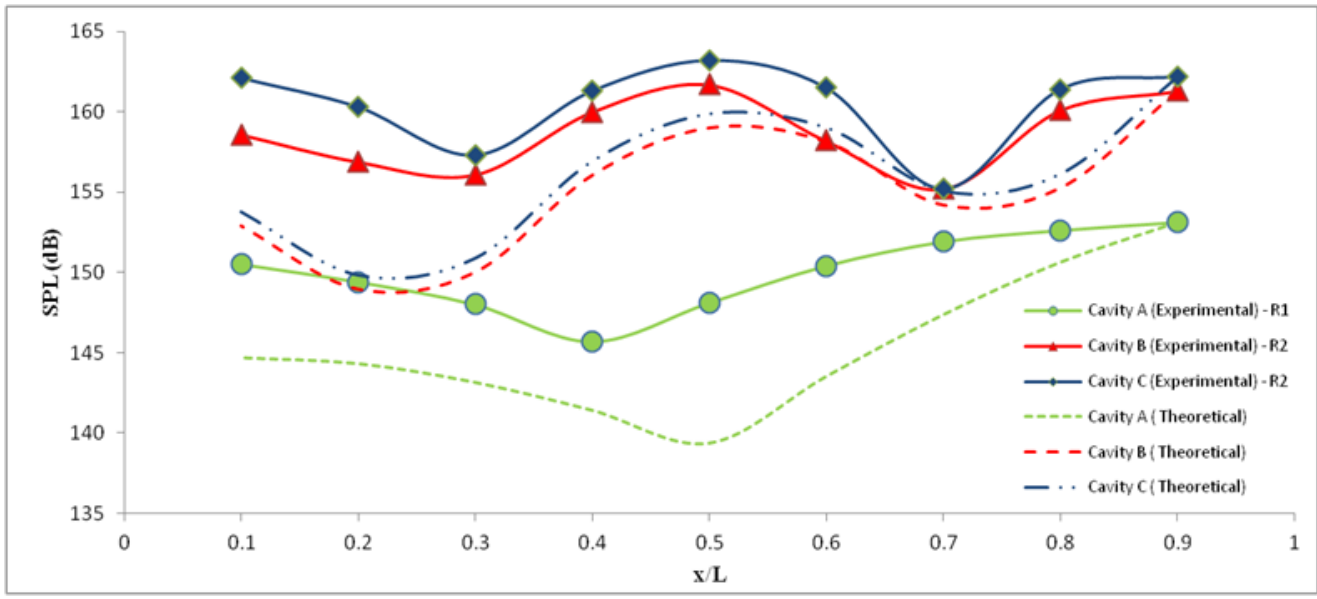

Figure 7. Experimental \& theoretical mode shapes of dominant modes of different cavities $(M=0.71)$ 


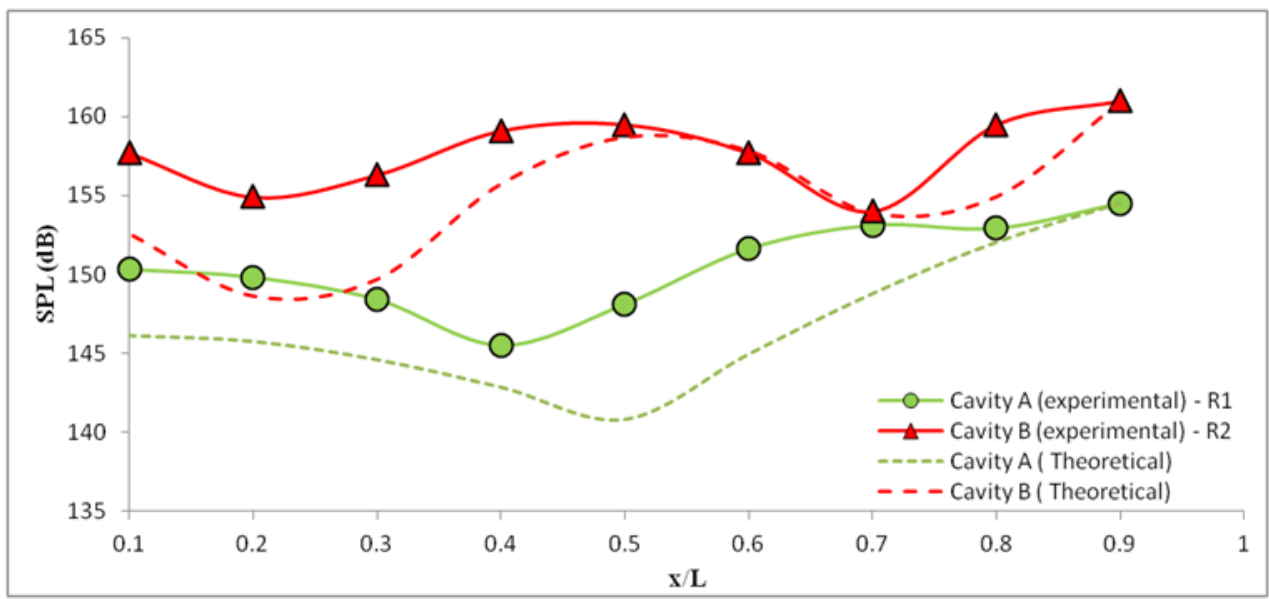

Figure 8. Experimental \& theoretical mode shapes of dominant modes of different cavities $(M=0.85)$

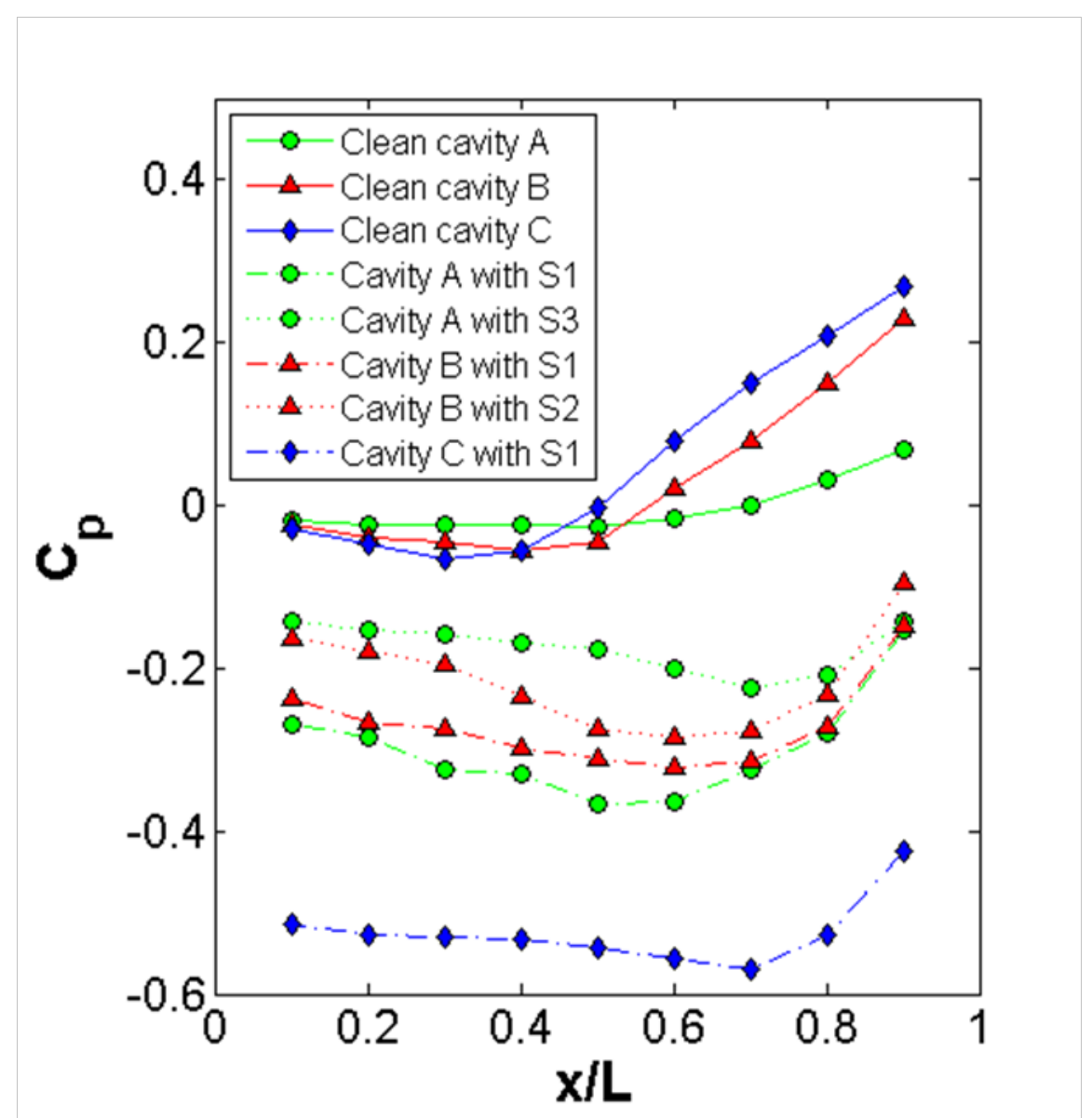

Figure 9. Effect of spoilers on cavity floor centreline mean pressure distribution $(M=0.71)$ 


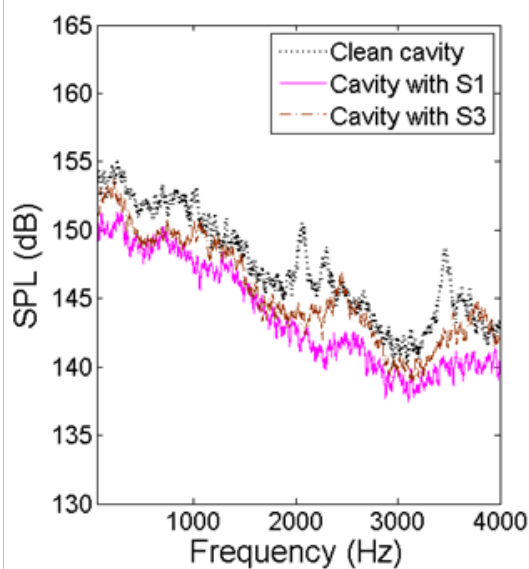

(a)

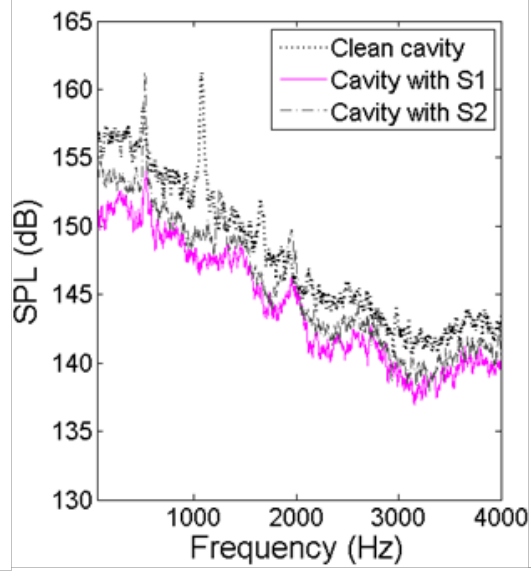

(b)

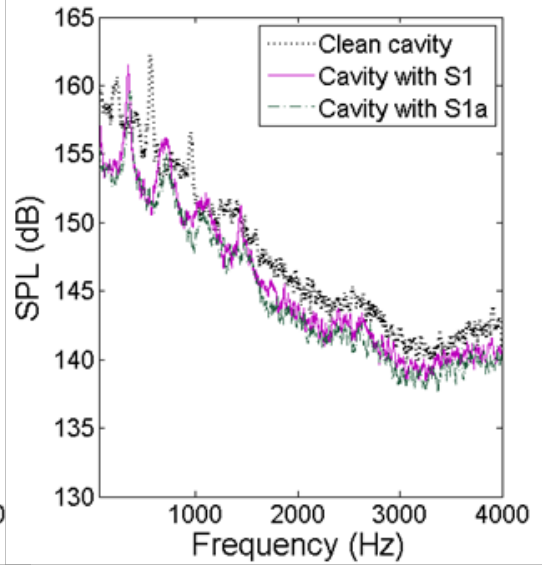

(c)

Figure 10. Effect of spoilers on power spectra at $x / L=0.9(M=0.71)$ for (a) cavity $A$, (b) cavity $B$ and

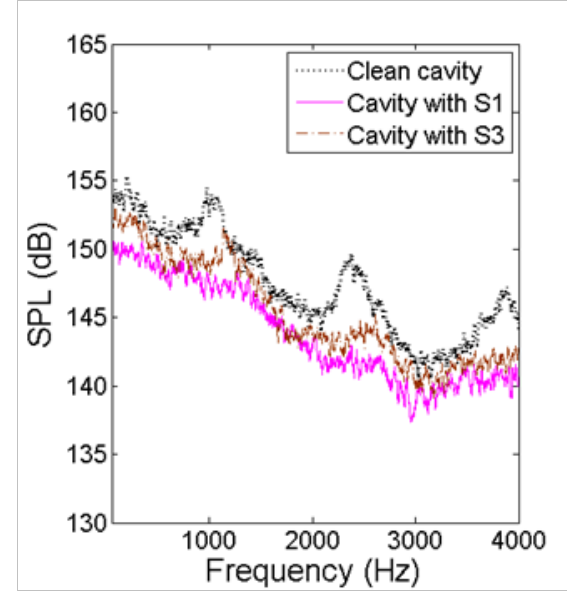

(a) (c) cavity $\mathbf{C}$

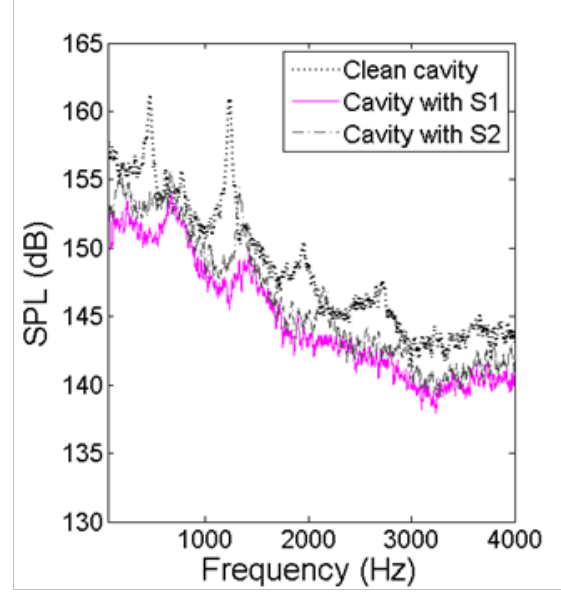

(b)

Figure 11. Effect of spoilers on power spectra at $x / L=0.9(M=0.85)$ for (a) cavity A and (b) cavity B

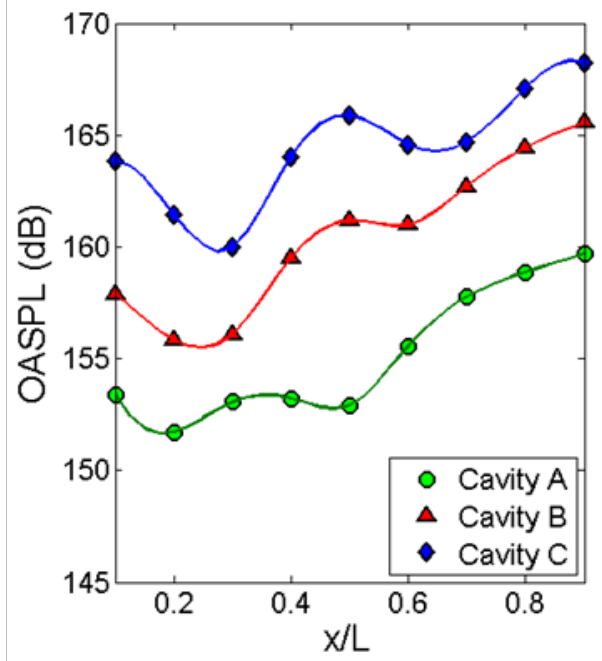

(a)

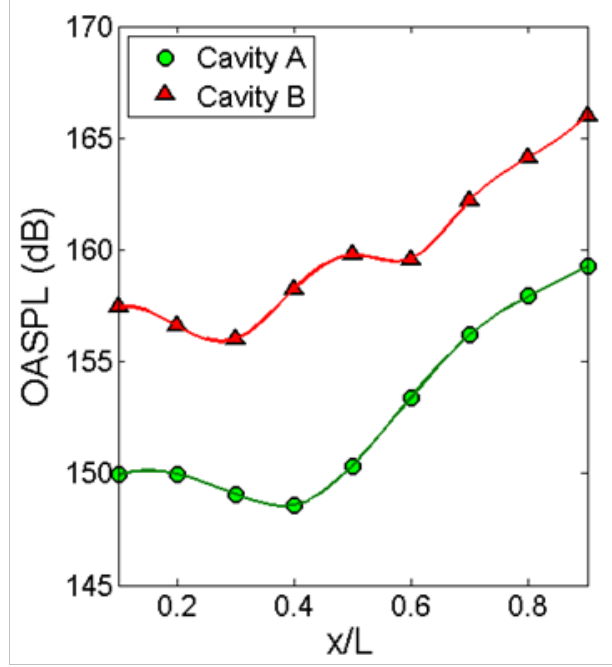

(b)

Figure 12. OASPL distribution on clean cavity floor centreline for (a) $M=0.71$ and (b) $M=0.85$ 


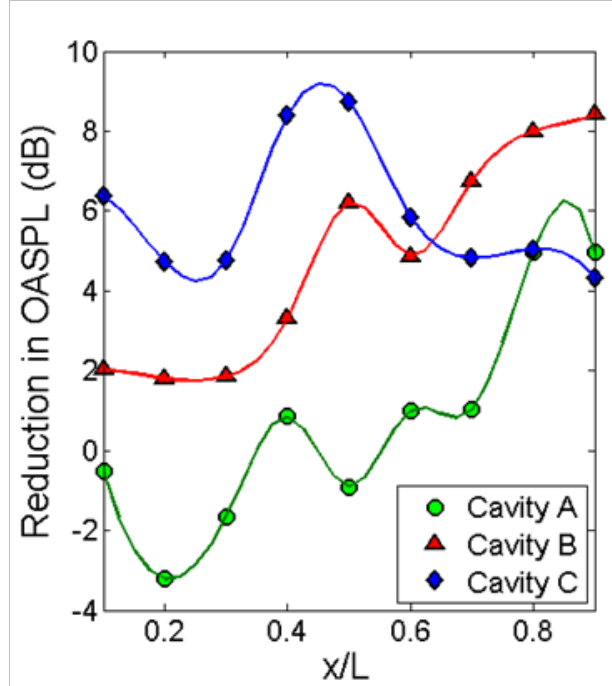

(a)

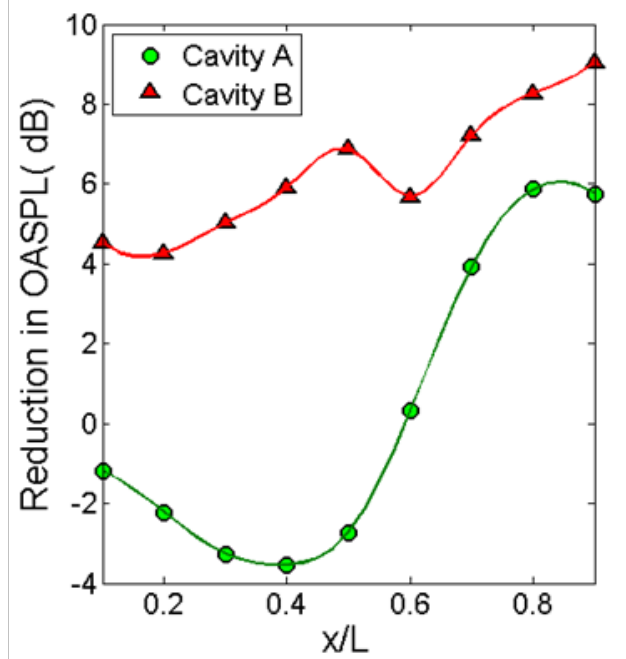

(b)

Figure 13. Centreline OASPL reduction for different cavities with spoiler $S 1$ at (a) $M=0.71$ and (b) $M=0.85$

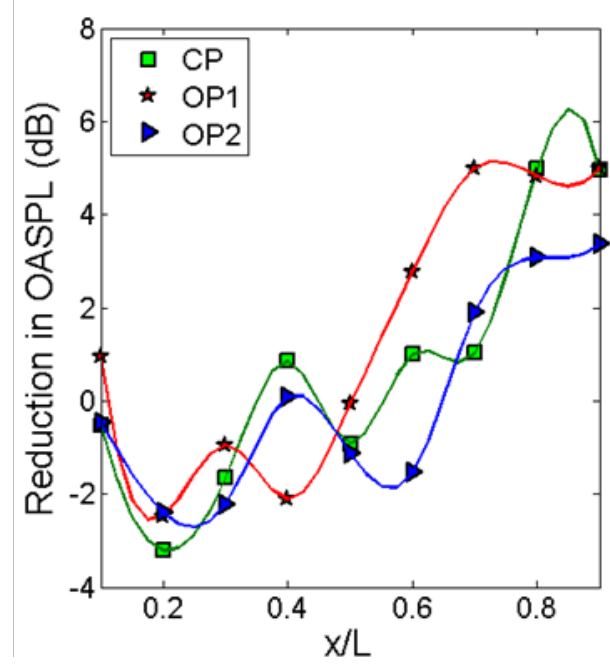

Figure 14. OASPL reduction for cavity A with spoiler $\mathrm{S1}(\mathrm{M}=\mathbf{0 . 7 1})$

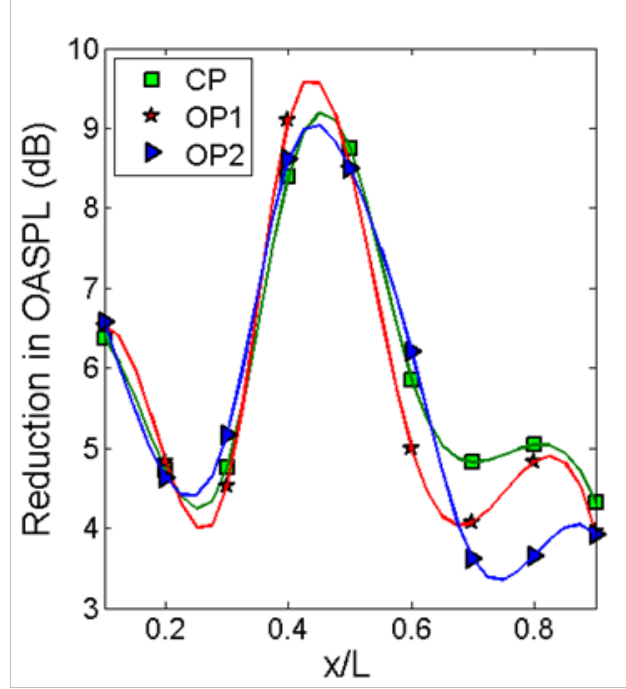

Figure 16. OASPL reduction for cavity $C$ with spoiler $S 1(M=0.71)$

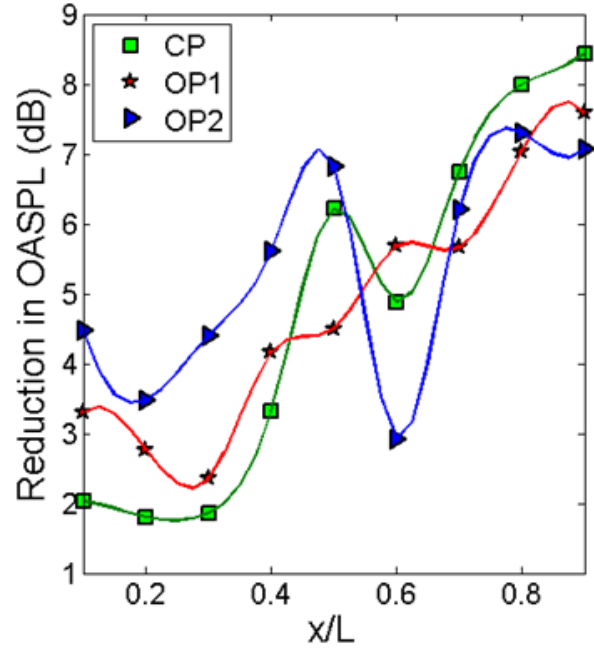

Figure 15. OASPL reduction for cavity $B$ with spoiler $S 1(M=0.71)$

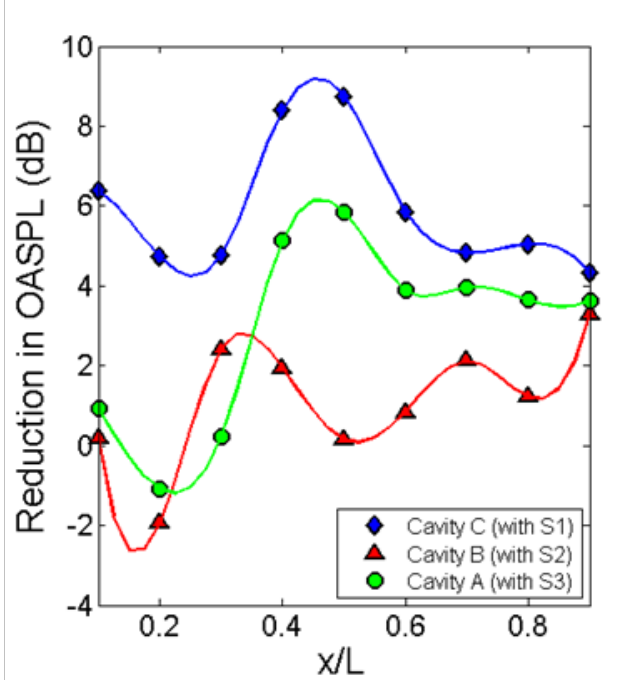

Figure 17. OASPL reduction at $C P$ for constant $h / D=0.125$ 


\section{Conclusions}

An experiment designed to study the effect of scaling on cavity flow dynamics and control was performed by studying three cavities with different values of $\mathrm{L} / \delta$ viz. cavity A $(\mathrm{L} / \delta=10.35)$, cavity $\mathrm{B}(\mathrm{L} / \delta=20.7)$ and cavity $\mathrm{C}$ $(\mathrm{L} / \delta=41.4)$. The cavities had $\mathrm{L} / \mathrm{D}=5$ and $\mathrm{L} / \mathrm{W}=2$ and the flow Mach numbers of the experiments were 0.71 (for cavities A, B and C) and 0.85 (for cavities A and B).

Power spectral analysis based on unsteady pressure measurements revealed peaks of high amplitude in the frequency spectra. The peaks generally corresponded to the Rossiter frequencies and thus the cavity oscillations followed the feedback mechanism explained by Rossiter. The amplitude of the Rossiter frequencies decreased with decrease in $\mathrm{L} / \delta$ and, for cavity $\mathrm{A}$, the spectrum was laden with broadband with feebly prominent peaks at $\mathrm{M}=0.71$. The peaks for cavity A, however, became more prominent for $\mathrm{M}=0.85$. Time-frequency analysis showed the occurrence of mode-switching for cavities $\mathrm{B}$ and $\mathrm{C}$ at $\mathrm{M}=0.71$.

The effect of a leading-edge control device on these different scales was also studied. The leading-edge device selected for the purpose was a sawtooth spoiler. The introduction of a spoiler with height approximately equal to the boundary layer thickness led to attenuation of cavity tones and decrease in broadband level in most of the measurement points. However, there was a significant difference in the attenuation values observed in the different scales. At $\mathrm{M}=0.71$, the maximum reduction in tonal amplitudes was $12.7 \mathrm{~dB}$ for cavity $\mathrm{B}$ at $\mathrm{x} / \mathrm{L}=0.9$ and the maximum OASPL reduction was $8.7 \mathrm{~dB}$ for cavity $\mathrm{C}$ at $\mathrm{x} / \mathrm{L}=0.5$. In general, it can be concluded from the results that the reduction in OASPL increased with the increase in L/ $\delta$ for the first $60 \%$ of the length of the cavity and the trend changed thereafter. The reduction in OASPL is highly dependent on the acoustic signature of the clean cavity, which in turn is dependent on the scale of the model.

The reduction in OASPL showed streamwise and spanwise variations. In the streamwise direction, it was seen that for cavities with very intense oscillations, the variation of reduction value approximately followed the mode shape of the dominant tone. For cavities B and C, the reduction at a particular point was related to the amplitude of the dominant tone present there. However, for cavity $\mathrm{A}$ at $\mathrm{M}=0.71$, the trend was disturbed due to the absence of steep peaks in the clean case and due to the increasing influence of the shear layer turbulence on the cavity floor. 
In the spanwise direction, the variation of the OASPL reduction was influenced by the three-dimensional shear layer structures emerging after the spoiler. The shear layer coherence was found to be highest for the cavity with highest $\mathrm{L} / \delta$ value. The values at a particular longitudinal (XY) plane were affected by the 'peaks' and 'valleys' of the sawtooth structures in the spoiler. Cavity C, however, was found to be immune to this effect. It was also observed that the reduction of OASPL decreased with the decrease in the height of the spoiler.

From the observations above, it is hypothesized that lifting of the shear layer and disruption of the spatial coherence of the shear layer is responsible for the attenuation of cavity tones that has been observed. The performance of the leading-edge device is highly sensitive to the scale of the model, which decides the acoustic character of the clean cavity. Also, it was seen that, although OASPL levels were reduced, the spoilers were not successful in completely eliminating the tones for cavity C. The suppression values achieved are sufficient to control the unsteadines of smaller scale models but for a bigger scale, the cavity unsteadiness increases so much that the suppression values achieved by the spoilers were insufficient to cope with it. Although only spoilers have been used for this study, the implications are generic and most of the leading-edge devices would be expected to suffer the same setbacks with increasing scale as the spoilers. This is because the fluid dynamic mechanism that most of the leading-edge devices induce is focussed at 'breaking' the feedback loop in two points :

1. the point where the feedback wave inside the cavity interact with the shear layer and

2. the point where the shear layer interacts with the aft wall

The disruption at either of the two points weakens the feedback mechanism and decreases the amplitude of the cavity tones. Weakening the feedback loop at these two points, however, has a serious drawback when it comes to scaling the cavity, as was seen in this study. The interaction between the shear layer and aft wall gets strengthened with increase in scale due to amplification of shear layer in the downstream direction. This was clearly observed in the power spectra of cavity C.

The results obtained from the studies highlight the fact that although clean cavities of different scales follow the same Rossiter mechanism for the generation of tones, there are interesting variations in certain spectral characteristics. This underscores the significant role played by the factor $\mathrm{L} / \delta$ in cavity oscillations. The current studies also show that the efficiency of the leading-edge spoiler in suppressing oscillations at a particular cavity 
floor location is more sensitive to the scale of the model than to the Mach number. The successful implementation of a spoiler depends both on its height with respect to the incoming boundary layer and with respect to the size of the cavity. Therefor it is recommended that before implementing a passive control device for practical use, the device should be tested in the possible range of $L / \delta$ that may be experienced in flight.

\section{Acknowledgments}

We would like to thank Dr. Mark Finnis (Principal Research Officer) and Mr. David Wasley (Senior Laboratory Manager) of Cranfield University's Aeromechanical Systems Group for their valuable help in the setting up of the experiments and wind tunnel.

\section{References}

[1] Shaw, L., Clark, R. and Talmadge, D., "F-111 generic weapons bay acoustic environment", Journal of Aircraft, Vol. 25, No. 2, 1988, pp. 147-153.

[2] Cattafesta III, L. N., Williams, D. R., Rowley, C. W. and Alvi, F., "Review of active control of flow-induced cavity resonance", 33 ${ }^{\text {rd }}$ AIAA Fluid Dynamics Conference, Orlando,FL. Paper 2003-3567, June 2003.

[3] Lawson, S. J. and Barakos, G. N., "Assessment of passive flow control for transonic cavity flow using detached-eddy simulation", Journal of Aircraft, Vol. 46, No. 3, 2009, pp. 1009-1029.

[4] Ahuja, K. K. and Mendoza, J., "Effects of cavity dimension, boundary layer and temperature on cavity with emphasis on benchmark data to validate computational aeroacoustic codes", NASA TR 4653, 1995, pp 6578.

[5] Ross, J., "High speed acoustic measurements in cavities", Report No. F49620-98-1-0167, DERA, UK, 2001.

[6] Rossiter, J. E., "Wind tunnel experiments on the flow over rectangular cavities at subsonic and transonic speeds", Royal Aircraft Establishment TR 64037, Bedford, UK.., Oct 1964 
[7] Ukeiley, L. S., Ponton, M. K., Seiner, J. M. and Jansen, B., "Suppression of pressure loads in cavity flows", AIAA Journal, Vol. 42, No. 1, 2004, pp. 70-79.

[8] Ashworth, R., "DES of a cavity with spoiler", Notes on Numerical Fluid Mechanics, Vol 97, 2008, pp. 162171.

[9] Khanal, B., Knowles,K. and Saddington,A,J., "Computational investigation of cavity flow control using a passive device", Aeronautical Journal, Vol. 116, No.1176, 2008, pp 153-174

[10] Heller, H. H., Holmes, D. G. and Covert, E. E., "Flow-induced pressure oscillations in shallow cavities", Journal of Sound and Vibration, Vol. 18, No. 4, 1971, pp 545-553

[11] Kegerise, M. A., Spina, E. F., Garg, S. and Cattafesta, L. N., "Mode-switching and nonlinear effects in compressible flow over a cavity", Physics of Fluids, Vol. 16, No. 3, 2004, pp. 678-687.

[12] Plumblee, H. E., Gibson, J. S. and Lassiter, L. W., "A theoretical and experimental investigation of the acoustic response of cavities in an aerodynamic flow", WADD-TR-61-75,1962, Wright Patterson AirForce Base, Dayton, Ohio., 1962.

[13] Bauer, R. C. and Dix, R. E., "Engineering model of unsteady flow in a cavity", TR-91-17, Arnold Engineering Development Centre, 1991.

[14] Clarke, R. L., Kaufman, L. G. and Maciulaitis, A., " Mach 0.6 - 3.0 Flows Past Rectangular Cavities", Airforce Wright Aero Lab Report. AFWAL TR-82-3112, 1983.

[15] Heller, H. H. and Bliss, D. B., "The Physical Mechanism of Flow-Induced Pressure Fluctuations in Cavities and Concepts of Their Suppression", $2^{\text {nd }}$ Aeroacoustics Conference, Hampton, VA. Paper 75-491, March 1975 
[16] Gloerfelt, X., Bogey, C. and Bailly, C., "Numerical investigation of the coexistence of multiple tones in flow-induced cavity ", Int. J. of Aeroacoustics, Vol. 2, No. 2, 2003, pp. 99-124.

[17] Smith, D. and Shaw, L., "Prediction of the pressure oscillation in cavities exposed to aerodynamic flow", AFFDL-75-34, Air Force Flight Dynamics Laboratory, Wright - Patterson Air Force- Base, Dayton, Ohio., 1975 